\title{
Observations of Stars and Stellar Systems with the Navy Prototype Optical Interferometer
}

\author{
Thomas A. Pauls \\ Code 7210, Naval Research Laboratory, Washington, DC 20375
}

\begin{abstract}
We present results of observational programs with the Navy Prototype Optical Interferometer (NPOI) in astrometry, the study the angular diameters of individual stars, and the measurement of binary star systems. These observations include measurements of the closure phase, which allow us to make images of simple systems.
\end{abstract}

\section{Introduction}

The Navy Prototype Optical Interferometer (NPOI), shown in Figure 1, is located on Anderson Mesa near Flagstaff, Arizona, at an altitude of $2200 \mathrm{~m}$, and is described in detail by Armstrong et al. (1998a). We will only give a brief overview here. The instrument consists of two subarrays of siderostats, in an equianglar and equilateral $\mathrm{Y}$ configuration, which share common components. Four siderostats in permanent enclosures form the astrometric array, while six moveable siderostats form the aperture synthesis imaging array. Data is collected in 32 channels covering the range from $450 \mathrm{~nm}$ to $850 \mathrm{~nm}$. The longest baseline is $437 \mathrm{~m}$, and this combined with the shortest wavelength gives a resolution limit of $200 \mu$ as. The instrument tracks atmospheric phase fluctuations in real time and uses phase bootstrapping (Armstrong et al. 1998b) to improve the signal-to-noise ratio on long baselines. The results reported here were made using three of the astrometric stations, with baseline lengths of 19,22, and 38 meters. The NPOI is a joint program of the Naval Research Laboratory, the U. S. Naval Observatory, and the Lowell Observatory. In this paper we will review current scientific results obtained with the NPOI, and discuss plans for the future.

\section{Astrometry}

The initial goal of the NPOI astrometry program is to produce accurate positions for about 1000 of the brighter stars in the Hipparcos catalog, with an internal accuracy of $1-3$ mas. The positional accuarcy of the Hipparcos reference frame will by degraded to about 10 mas by 2001 due to proper motions. Secondary goals include long time-base proper motion studies, and investigating the Hipparcos catalog for zonal errors. Recently, work has concentrated on corrections for atmospheric dispersion, which has reduced the r.m.s. fluctations of stellar delays to $\sim 500 \mathrm{~nm}$. Baseline measurements are now repeatable at a level of $\sim 200 \mathrm{~nm}$ (Hutter 2000). 


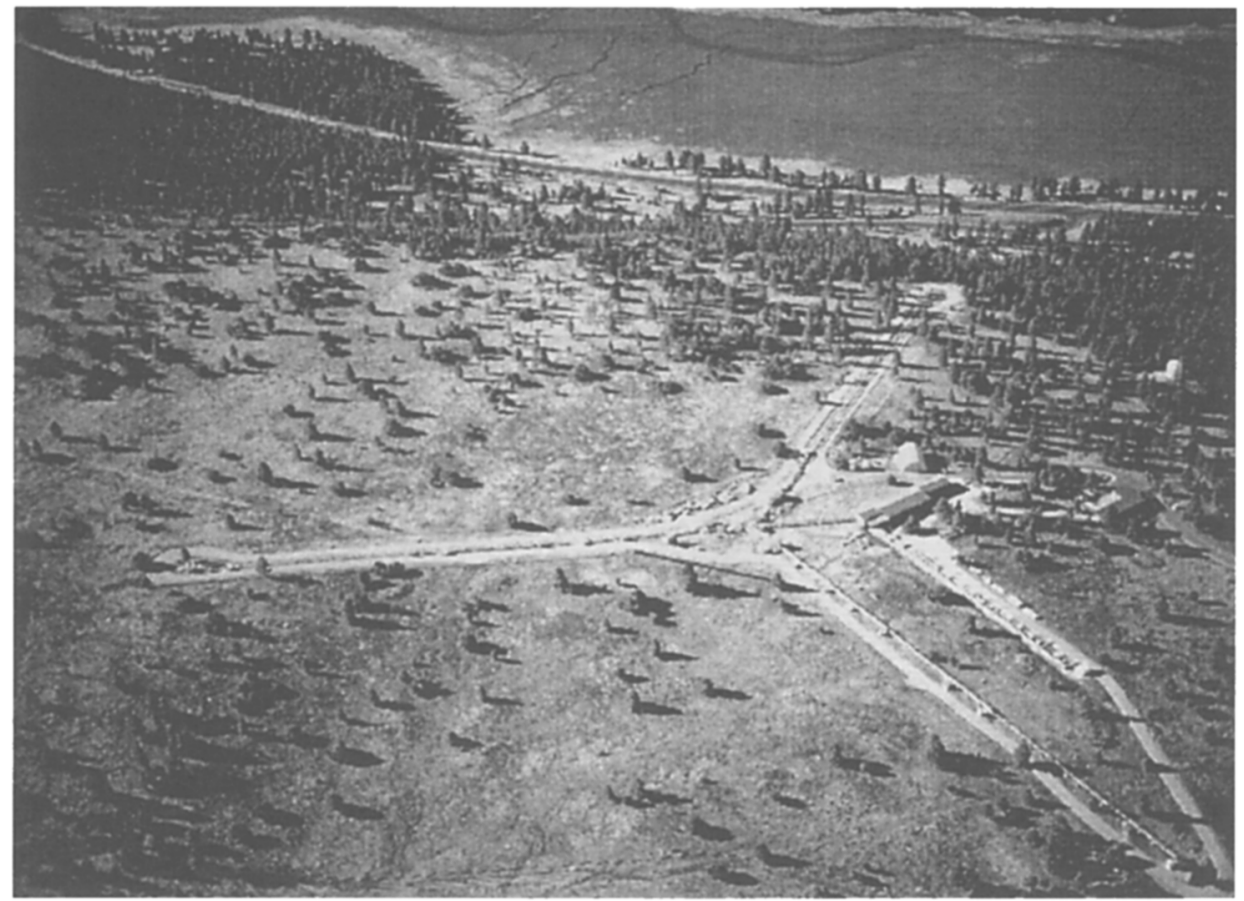

Figure 1. Aerial view of the Navy Prototype Optical Interferometer on Anderson Mesa near Flagstaff, Arizona. The north arm of the array extends from the center to the lower right corner of the picture. The four astrometric huts are at the center of the array. The pipes along the arms are for the vacuum feed system which brings light from the siderostats to the optics laboratory, which is the long building to the right of the array center. 


\section{Stellar Diameters and Effective Temperatures}

The NPOI stellar diameters program is designed to test current theoretical models of stellar atmospheres by comparing measured diameters with those predicted by the models. This comparison is a valuable check on the stellar effective temperature scale. For example, the infrared flux method is one of the most reliable ways to estimate the $T_{\text {eff }}$ for stars. The method uses the ratio of the apparent bolometeric flux, $f_{b o l}$, to the apparent flux in an infrared bandpass, $f_{K}$. Theoretical models predict the behavior of $\left[f_{b o l} / f_{K}\right]$ as a function of $T_{e f f}$, and can be used to predict limb darkened angular diameters, $\theta_{L D}$ of stars since $\theta_{L D}^{2} T_{e f f} \propto f_{b o l}$. The current observing program tests the predicted and measured limb-darkened diameters of $\mathrm{K}$ stars of different luminosity classes from supergiants to dwarfs. Results so far show very close agreement for giants, the agreement is less good for supergiants, and there appears to be real disagreement in the case of dwarfs, with the measured diameters larger that predicted by approximately $10 \%$. The details of this work can be found in Hajian et al. (1998) and Pauls et al. $(1998,2000)$, and references therein.

One of the long-standing goals of stellar diameter measurements has been to detect the change in the diameter of a cepheid variable star. Although the current baselines make such a measurement difficult, considerable effort has been expended on a study of the star $\delta$ Cep (Armstrong et al. 2001, Nordgren et al. 2000). The results to date are consistant with a marginal detection of a diameter change that is in phase with the optical variations.

\section{Binary Stars}

Currently, NPOI has an extensive binary star program lead by Christian Hummel. The goal is to increase the number of stars with precisely measured masses and luminosities. This goal is being persued in three ways: first, by completing orbits partially measured with the Mark III interferometer in Mt. Wilson; second, observing new binaries from a selection of bright spectroscopic binaries from Batten catalog; and third, observing binaries from the Washington Double Star Catalog with separations within the range of the NPOI baselines. Early results on a sample of spectroscopic binaries can be found in Hummel (1998a). Other results include a detailed study of Mizar A (Hummel et al. 1998b), and the chance discovery that $\zeta$ Orionis is a double star with a fourth magnitude companion (Hummel et al. 2000).

\section{Aperture Synthesis Imaging}

The first science paper from the NPOI reported observations of the star Mizar A ( $\zeta^{1}$ Ursae Majoris), a known spectroscopic binary (Benson et al. 1997). The paper used the multispecatral capabilities of the NPOI along with the closure phases to make a simple image of the system using techniques developed for radio interferometry.

The NPOI measures the real and imaginary parts of the complex visibility on each baseline at each spectral channel. For optical interferometers (photon counting systems) it is the square of the visibility which is an unbiased estimator 
(see, for example, Armstrong et al. 1998a). Thus, each data set consists of the squared visibilities on each baseline at each spectral channel, along with the complex triple product, constructed directly from the measured real and imaginary parts of the complex visibility, for each closed triangle. The argument of the triple product is the closure phase for that triangle. The NPOI imaging array uses 6 siderostats, which results in 15 baselines and 12 closure phases. With 32 spectral channels this gives a theoretical total of 480 squared visibilities and 384 closure phases for each measurement. The challenge now is to develop calibration techniques and imaging algorithms for these rich new data.

\section{Acknowledgements}

The NPOI owes its success to the work of a large number of dedicated people over many years. NPOI is a joint project of the Naval Research Laboratory, the U. S. Naval Observatory, and the Lowell Observatory, and receives support from the Office of Naval Research and the Oceanographer of the Navy. More information can be found at http://ad.usno.navy.mil/npoi.

\section{References}

Armstrong, J. T., et al. 1998a, ApJ, 496, 550

Armstrong, J. T., et al. 1998b, Proc. SPIE, 3350, 461

Armstrong, J. T., et al. 2001, AJ, in press

Hajian A. R., et al. 1998, ApJ, 496, 484

Hummel, C. A. 1998a, Proc. SPIE, 3350, 483

Hummel, C. A. 1998b, AJ, 116, 2536

Hummel, C. A., et al. 2000, ApJ, 540, 91

Hutter, D. J. 2000 in JPL Pub. 00-009 07/00, Principles of Long Baseline Stellar Interferometry, ed. P. R. Lawson (Pasadena: JPL), 165

Nordgren, T. E., et al. 2000, ApJ, 543, 972

Pauls, T. A., et al. 1998, Proc. SPIE, 3350, 467

Pauls, T. A., et al. 2000, Proc. SPIE, 4006, 584 